\title{
Strong iron demand during hypoxia-induced erythropoiesis is associated with down-regulation of iron-related proteins and myoglobin in human skeletal muscle
}

\author{
Paul Robach, ${ }^{1,9}$ Gaetano Cairo, ${ }^{2}$ Cecilia Gelfi, ${ }^{3}$ Francesca Bernuzzi, ${ }^{2}$ Henriette Pilegaard, ${ }^{4}$ Agnese Viganò, ${ }^{3}$ \\ Paolo Santambrogio, ${ }^{5}$ Paolo Cerretelli, ${ }^{3}$ José A. L. Calbet, ${ }^{6}$ Stéphane Moutereau, ${ }^{7}$ and Carsten Lundby ${ }^{4,8}$ \\ 1'Département médical, Ecole Nationale de Ski et d'Alpinisme, Chamonix, France; '2Institute of General Pathology, University of Milano, Italy; ${ }^{3}$ Department of \\ Sciences and Biomedical Technologies, University of Milano, Segrate, Italy; ${ }^{4}$ The Copenhagen Muscle Research Centre and Centre of Inflammation and \\ Metabolism, Institute of Molecular Biology and Physiology, University of Copenhagen, Denmark; ${ }^{5}$ Protein Engineering Unit, Dibit, Instituto di Ricerca e Cura a \\ Carattere Scientifico (IRCCS) H.S. Raffaele, Milano, Italy; ${ }^{6}$ Department of Physical Education, University of Las Palmas de Gran Canaria, Spain; ${ }^{7}$ Laboratoire de \\ Biochimie, Hôpital Henri-Mondor, Créteil, France; ${ }^{8}$ Department of Sport Science, University of Århus, Denmark; 9 ${ }^{2}$ aboratoire "Réponses Cellulaires et \\ Fonctionnelles à l'Hypoxie," Association pour la Recherche en Physiologie de l'Environment (ARPE), Université Paris 13, Bobigny, France
}

\begin{abstract}
Iron is essential for oxygen transport because it is incorporated in the heme of the oxygen-binding proteins hemoglobin and myoglobin. An interaction between iron homeostasis and oxygen regulation is further suggested during hypoxia, in which hemoglobin and myoglobin syntheses have been reported to increase. This study gives new insights into the changes in iron content and iron-oxygen interactions during enhanced erythropoiesis by simultaneously analyzing blood and muscle samples in humans exposed to 7 to 9 days of high altitude hypoxia (HA).
\end{abstract}

HA up-regulates iron acquisition by erythroid cells, mobilizes body iron, and increases hemoglobin concentration. However, contrary to our hypothesis that muscle iron proteins and myoglobin would also be up-regulated during HA, this study shows that HA lowers myoglobin expression by $35 \%$ and down-regulates iron-related proteins in skeletal muscle, as evidenced by decreases in L-ferritin (43\%), transferrin receptor (TfR; $50 \%)$, and total iron content $(37 \%)$. This parallel decrease in L-ferritin and TfR in HA occurs independently of increased hypoxia-inducible factor 1 (HIF-1) mRNA levels and unchanged binding activity of iron regulatory proteins, but concurrently with increased ferroportin mRNA levels, suggesting enhanced iron export. Thus, in HA, the elevated iron requirement associated with enhanced erythropoiesis presumably elicits iron mobilization and myoglobin down-modulation, suggesting an altered muscle oxygen homeostasis. (Blood. 2007;109:4724-4731)

๑) 2007 by The American Society of Hematology

\section{Introduction}

Iron is an essential element, required as a cofactor for proteins managing oxygen transport such as hemoglobin and myoglobin. Accordingly, iron metabolism is tightly interconnected to oxygen homeostasis. Hypoxia, known to occur in a broad range of pathologic or environmental conditions, activates the hypoxiainducible factor 1 (HIF-1), which is a transcription factor that controls a wide range of genes involved in adaptations to low oxygen pressure. ${ }^{1,2}$ Several of these genes are involved in iron homeostasis. For instance, expression of the transferrin receptor (TfR), which mediates cellular iron uptake, was shown to be up-regulated in hypoxia through the HIF-1 pathway. ${ }^{3,4}$ Moreover, hypoxia was found to alter the activity of iron regulatory proteins (IRPs), cytoplasmic proteins which, according to intracellular iron availability, regulate iron metabolism by binding to conserved iron regulatory elements (IREs) in the untranslated regions of ferritin and TfR mRNAs. ${ }^{5,6}$ The inverse but coordinate regulation of the iron storage protein ferritin and the iron uptake protein TfR finely adjusts iron levels in order to provide an adequate level of this micronutrient while preventing its accumulation and toxicity. In addition, other proteins involved in cellular and systemic iron balance, such as ceruloplasmin, hepcidin, and ferroportin, may also be influenced by hypoxia. ${ }^{7-9}$
At the level of the whole organism, iron plays a key role in maintaining oxygen homeostasis in hypoxia. In the erythropoietic compartment, which contains approximately $70 \%$ of the body iron stores, hypoxia stimulates erythropoiesis and promotes hemoglobin synthesis. Because iron is a central component of heme, the higher heme production associated with exposure to hypoxia requires the entry of iron into the erythroid cell to be dramatically increased, which in turn may lead to inadequate iron availability for other body compartments during erythropoietic stimulation. ${ }^{10}$ As a consequence, body iron stores gradually decrease during prolonged exposure to hypoxia. ${ }^{11,12}$

Skeletal muscle is of great interest regarding iron metabolism, ${ }^{13}$ because it represents about $40 \%$ of body mass and contains $10 \%$ to $15 \%$ of body iron, which is mainly located in myoglobin, a hemoprotein known as an oxygen-storage protein and also as an oxygen-diffusion facilitator. ${ }^{14,15}$ Exposure to prolonged hypoxia may enhance the synthesis of myoglobin, ${ }^{16-21}$ therefore preserving muscle oxygen homeostasis. This anticipates that iron entry into muscle cells should be facilitated under hypoxic conditions in order to allow for increased iron incorporation in the heme moiety of myoglobin. However, this assumption implies that enough iron is available for skeletal muscle to enhance myoglobin synthesis at a
Submitted August 7, 2006; accepted January 26, 2007. Prepublished online as Blood First Edition Paper, February 20, 2007; DOI 10.1182/blood-2006-08040006.

An Inside Blood analysis of this article appears at the front of this issue.
The publication costs of this article were defrayed in part by page charge payment. Therefore, and solely to indicate this fact, this article is hereby marked "advertisement" in accordance with 18 USC section 1734.

(C) 2007 by The American Society of Hematology 
time when there is an increased iron demand from the enhanced erythropoiesis. It could be hypothesized that, during severe hypoxia, the capacity of skeletal muscle to take up and incorporate iron is increased despite a high erythropoietic iron requirement. Thus, the aim of this study was to determine the expression of proteins of iron metabolism and myoglobin content in muscle tissue during exposure to hypoxia in the face of the enhanced iron request for erythropoiesis. This was examined by evaluating the expression of iron and hemoproteins simultaneously in 2 distinct compartments (ie, in blood, the central erythropoietic compartment, and in skeletal muscle of healthy humans exposed to 7 to 9 days of high altitude hypoxia [HA]). The results of this study give new insights into the competition for iron between "peripheral and central compartments" under hypoxic conditions, providing novel information about the molecular aspects of iron-oxygen interactions.

\section{Materials and methods}

\section{Participants}

A total of 9 healthy male volunteers participated in the study. They were not acclimatized to altitude prior to the experiment. Their characteristics (means $\pm \mathrm{SD}$ ) were: age, $27 \pm 2$ years; height, $181 \pm 2 \mathrm{~cm}$; body mass, $79 \pm 8 \mathrm{~kg}$; and maximal oxygen uptake, $54 \pm 6 \mathrm{~mL} / \mathrm{min} / \mathrm{kg}$. Written informed consent to participate in the study was obtained from all participants. This study was carried out according to the Declaration of Helsinki and was approved by the Ethical Committee of Copenhagen and Frederiksberg Counties, Denmark.

\section{Procedures}

Sea-level procedure. The participants were first investigated at sea level (SL) in Copenhagen, Denmark. Before the measurements, the participants stayed for 3 consecutive days at the laboratory without performing any physical activity. Food intake was controlled during this 3-day period. On the fourth day (9:00-10:00 a.m.), arterial and venous blood samples were collected, and a muscle biopsy was obtained from the vastus lateralis muscle.

High-altitude procedure. The sea-level procedure was repeated under conditions of hypobaric hypoxia at the altitude of $4559 \mathrm{~m}$ (Capanna Regina Margherita, Monte Rosa, Italy). The average barometric pressure at $4559 \mathrm{~m}$ was $453 \mathrm{mmHg}$. The profile of exposure to hypoxia was as follows: the first day consisted of transport by cable car from $1300 \mathrm{~m}$ to $3200 \mathrm{~m}$, followed by a 2-hour climb to an altitude of $3611 \mathrm{~m}$ (Capanna Gnifetti), where the subjects stayed overnight (day 1). The next day, the subjects stayed inactive for 24 hours at $3611 \mathrm{~m}$ (day 2) in order to minimize the risk of acute mountain sickness upon arrival to $4559 \mathrm{~m}$. On the morning of the third day, after a 5-hour climb, they reached the altitude of $4559 \mathrm{~m}$ (day 3). The subjects spent the next 3 to 5 days in the high-altitude research laboratory (days 4 to 8). During this phase, the subjects were not physically active and the dietary intake was again controlled, being exactly the same as during the 3-day examination at sea level. On days 7 to 9 at $4559 \mathrm{~m}$, high-altitude measurements took place (blood samples and biopsy), with 3 participants evaluated each day, always in the morning (9:00-10:00 a.m.). The duration of exposure to hypoxia (7-9 days) was chosen according to previous data, indicating that during this period there is a high rate of erythropoiesis, along with a significant decrease in body iron stores. ${ }^{11}$

In summary, muscle biopsies and blood samples were obtained simultaneously on 2 separate occasions, first in normoxia at SL and second during HA.

Blood samples. Venous blood was sampled during supine rest from a forearm vein just prior to the muscle biopsy. Blood samples were allowed to clot at $4{ }^{\circ} \mathrm{C}$, centrifuged, and the serum was then stored at $-20^{\circ} \mathrm{C}$ for later analysis. Arterial and femoral venous blood was obtained from the right femoral artery and femoral vein, respectively, for immediate determination of blood gases, as previously described. ${ }^{22}$
Muscle biopsies. Under local anesthesia (lidocaine), a resting muscle biopsy was obtained from the middle portion of the vastus lateralis muscle by using the percutaneous needle biopsy technique with suction. The muscle specimen, on average of $90 \mathrm{mg}$, was immediately frozen in liquid nitrogen and stored at $-80^{\circ} \mathrm{C}$ for subsequent analysis.

\section{Blood analyses}

Arterial blood was sampled anaerobically in heparinized syringes and was immediately analyzed for hemoglobin concentration ([Hb]), oxygen saturation $\left(\mathrm{SaO}_{2}\right), \mathrm{CO}_{2}\left(\mathrm{PaCO}_{2}\right)$, and $\mathrm{O}_{2}\left(\mathrm{PaO}_{2}\right)$ partial pressures (ABL700; Radiometer, Copenhagen, Denmark). Simultaneously, femoral venous blood was sampled for determination of $\mathrm{O}_{2}$ partial pressure $\left(\mathrm{PfvO}_{2}\right)$. Arterial blood oxygen content $\left(\mathrm{CaO}_{2}\right)$ was computed from the oxygen saturation and $[\mathrm{Hb}]$; that is, $\left(1.34 \times[\mathrm{Hb}] \times \mathrm{SaO}_{2}\right)+\left(0.003 \times \mathrm{PaO}_{2}\right)$.

Serum concentration of erythropoietin (EPO) was assessed with an enzyme-linked immunosorbent assay (ELISA) kit (R\&D Systems, Minneapolis, MN). The serum concentrations of soluble transferrin receptor (sTfR), ferritin, transferrin, and ceruloplasmin were determined by nephelometry (Behring Nephelemeter Analyser II; Dade Behring GmbH, Marburg, Germany). The reagents (Dade Behring) used for those tests were $\mathrm{N}$ latex sTfR, $\mathrm{N}$ latex ferritin, human transferrin, and human ceruloplasmin, respectively. The serum iron concentration was assessed by ferrozin colorimetry with an iron reagent (Advia1650; Bayer Diagnostic, Dublin, Ireland).

\section{Muscle biopsy analyses}

Protein analyses. Muscle tissue was homogenized manually using a glass-glass tissue homogenizer in $10 \mathrm{mM}$ HEPES (pH 7.6), $3 \mathrm{mM} \mathrm{MgCl}_{2}$, $40 \mathrm{mM} \mathrm{KCl}, 5 \%$ glycerol, $0.2 \%$ Nonidet P40, $1 \mathrm{mM}$ DTT, and a mixture of protease inhibitors (Sigma Chemical, Milano, Italy). Lysates were incubated on ice for $30 \mathrm{~min}$, and debris was pelleted by centrifugation at $14500 \mathrm{~g}$ at $4^{\circ} \mathrm{C}$. The supernatant was aliquoted and stored at $-80^{\circ} \mathrm{C}$. Protein concentration was determined using the Dc protein assay (BioRad, Segrate, Italy).

For immunoblot analyses, equal amounts of protein were resolved by SDS-PAGE and transferred onto PVDF membranes (Amersham Biosciences, Milano, Italy). Antigens were detected using mouse monoclonal anti-TfR1 (Zymed, San Francisco, CA) and anti- $\alpha$-tubulin (clone B-5-1-1; Sigma Chemical) antibodies. Primary antibodies were revealed with horseradish peroxidase (HRP)-conjugated secondary antibodies (Amersham Biosciences) and a chemiluminescence kit (ECL; Amersham Biosciences).

Ferritin concentrations were determined in cell lysates by means of an ELISA assay based on monoclonal antibodies specific for human $\mathrm{H}$ - and L-ferritin subunits (rH02 and LF03) and calibrated with the corresponding recombinant homopolymers. ${ }^{23}$ The specificity and absence of crossreactivity of the antibodies have been previously described. ${ }^{23}$ The microtiter plates were coated with $1 \mu \mathrm{g}$ of a polyclonal antibody specific for mouse $\mathrm{H}$ or L-ferritin. Soluble tissue homogenates or standard ferritins were diluted in $50 \mathrm{mM} \mathrm{Na}$ phosphate ( $\mathrm{pH} 7.4$ ), $150 \mathrm{mM} \mathrm{NaCl}, 0.05 \%$ (vol/vol) Tween-20, and $1 \%$ bovine serum albumin, and added to the plates. The presence of ferritin was revealed by incubation with the same antibody labeled with HRP. Peroxidase activity was developed with o-phenylenediamine dihydrochloride (Sigma Chemical).

RNA electromobility shift assay (EMSA) experiments were performed using $5 \mu \mathrm{g}$ of muscle protein lysate and a molar excess of ${ }^{32} \mathrm{P}$-labeled human $\mathrm{H}$-ferritin iron regulatory element (IRE) probe, ${ }^{24}$ as previously described. ${ }^{25}$ Samples were resolved on $6 \%$ nondenaturing polyacrylamide gels, and RNA-protein complexes were visualized by autoradiography and quantitated by means of direct nuclear counting using an InstantImager (Packard Instruments, Milano, Italy).

For myoglobin analysis, equal amounts of protein extracts from each participant before and after hypoxia exposure were resuspended in lysis buffer ( $\mathrm{pH}$ 8.5) containing $7 \mathrm{M}$ urea, $2 \mathrm{M}$ thiourea, 4\% CHAPS, $65 \mathrm{mM}$ DTT, $0.5 \%$ immobilized $\mathrm{pH}$ gradient (IPG) buffer, $30 \mathrm{mM}$ Tris, and $1 \mathrm{mM}$ PMSF and separated on $18 \mathrm{~cm}$ IPG strips (6-11 pH gradient) with a voltage 
gradient from 200 to $8000 \mathrm{~V}$ using an IPG Phor electrophoresis unit (GE Healthcare, Bucks, United Kingdom). ${ }^{26}$ Prior to the second dimension, IPG strips were equilibrated for $15 \mathrm{~min}$ in an SDS equilibration/reduction buffer (6 M urea, 2\% SDS, 20\% glycerol, $374 \mathrm{mM}$ Tris- $\mathrm{HCl}$ [pH 8.8], and $65 \mathrm{mM}$ DTT), then alkylated by re-equilibrating for 8 minutes in the same buffer containing $135 \mathrm{mM}$ iodoacetamide instead of DTT. The second dimension separation used $20 \times 25 \mathrm{~cm}$ SDS gel $(12 \% \mathrm{~T})$. Electrophoresis of 12 gels was performed simultaneously at $15 \mathrm{~mA}$ overnight until the blue dye reached the bottom on an Ettan Dalt 12 system (GE Healthcare). Gels were stained with SYPRO Ruby (Molecular Probes, Leiden, the Netherlands) and images acquired by a Typhoon 9400 laser scanner (GE Healthcare) according to the manufacturer's recommendations (610 BP emission filter and 532 laser green). Spot quantification was performed by Image Quant Software (GE Healthcare). Protein extracts from each participant before and after hypoxia exposure were run in triplicate using 3 different gel batches.

RNA isolation, RT, and PCR. Total RNA was extracted from 20 to 25 $\mathrm{mg}$ of muscle by a modified guanidinium thiocyanate-phenol-chloroform extraction method adapted from Chomczynski and Sacchi, ${ }^{27}$ as previously described. ${ }^{28}$ Reverse transcription (RT) was performed using the Superscript II RNase $\mathrm{H}^{-}$system with Oligo dT (Invitrogen, Taastrup, Denmark), as previously described. ${ }^{28}$

The mRNA content of TfR, HIF- $1 \alpha$, HIF- $2 \alpha$, and ferroportin was determined by fluorescence-based real-time polymerase chain reaction (PCR; ABI PRISM 7900 Sequence Detection System; Applied Biosystems, Foster City, CA). Forward primers (FPs) and reverse primers (RPs) and TaqMan probes were designed from human-specific sequence data (EntrezNIH and Ensembl; Sanger Institute, Cambridge, United Kingdom) using computer software (Primer Express; Applied Biosystems). A total of 1 oligonucleotide from each set was designed to span an exon-exon junction, to avoid amplifying genomic DNA. The following sequences were used to amplify a fragment of TfR: FP, 5' GAATCCCAGCAGTTTCTTTCTGTT 3', RP, 5' TCAGTTCCTTATAGGTGTCCATGGT 3', and probe, 5' GTACCCAAATAAGGATAATCTGTGTCCTCGCAA $3^{\prime}$; HIF-1 $1 \alpha$ : FP, $5^{\prime}$ GCCCCAGATTCAGGATCAGA 3', RP, 5' TGGGACTATTAGGCTCAGGTGAAC $3^{\prime}$, and probe, $5^{\prime}$ ACCTAGTCCTTCCGATGGAAGCACTAGACAA 3'; HIF-2 $\alpha$ : FP, 5' GCCACCCAGTACCAGGACTACA 3', RP, 5' CCTCACAGTCATATCTGGTCAGTTCG $3^{\prime}$, and probe, 5' TCAGCCCACAAGGTGTCAGGCATG 3'; and ferroportin: FP, 5' TGACCAGGGCGGGAGA 3', RP, 5' GAGGTCAGGTAGTCGGCCAA 3', and probe, 5' CACAACCGCCAGAGAGGATGCTGTG $3^{\prime}$.

The probes were labeled with 5' 6-carboxyfluorescein (FAM) and 3' 6-carboxy-N, N, N', N'-tetramethylrhodamine (TAMRA). Prior optimization was conducted, as previously described. ${ }^{29}$ PCR amplification of each of the 4 target genes was performed (in triplicates) in a total reaction volume of $10 \mu \mathrm{L}$, as previously described. ${ }^{30}$ Serial dilutions were made from a representative pooled sample, and these samples were amplified together with the unknown samples and used to construct a standard curve by which the cycle threshold (Applied Biosystems) of each sample was converted to a relative amount. For each gene, the arbitrary target gene mRNA content was normalized to the cDNA content (see next paragraph) giving an mRNA expression ratio.

The amount of RNA/cDNA hybrids was determined in each sample using the PicoGreen reagent (Molecular Probes) and used as a measure of the cDNA content of each RT sample, as recently described. ${ }^{30}$ In brief, samples were run in triplicates in a 96-well white microplate (Thermo Labsystems, Vantaa, Finland) with a $2.5-\mu \mathrm{L}$ cDNA template, $100 \mu \mathrm{L}$ diluted PicoGreen, and a total reaction volume of $200 \mu \mathrm{L}$ in each well. The samples were analyzed in a fluorescence microplate reader (Fluorskan Ascent; Thermo Labsystems). The mean reading of each triplicate was converted to an absolute amount by the use of a standard curve constructed from a serial dilution of a bacteriophage lambda DNA standard (ranging from $1 \mathrm{ng}$ to $10 \mathrm{ng}$; Molecular Probes) run together with the samples on each plate.

Muscle total iron content. Total iron content in muscle tissue was determined chemically by atomic absorption spectrometry, as described by Pietrangelo et al. ${ }^{31}$

\section{Statistics}

The effect of high-altitude exposure on the different parameters ( 2 repeated measures) was evaluated with the Wilcoxon test. Before performing the statistical analysis, the mRNA data were logarithmically transformed. Statistics were done with the Statview Version 5.0. (SAS Institute, Cary, NC). Differences were considered significant at a $P$ level below .05 .

\section{Results}

\section{High altitude (HA) exposure stimulates iron incorporation in the erythropoietic tissue}

The response of the erythroid tissue in HA was first evaluated by analyzing blood parameters. Table 1 shows the typical hematologic response to a 7- to 9-day exposure to high-altitude hypoxia (ie, a decrease in both arterial oxygen partial pressure and oxygen saturation, counterbalanced by a $16 \%$ rise in hemoglobin concentration, resulting in unchanged arterial oxygen content). Stimulated erythropoiesis is indicated by the $40 \%$ increase in serum EPO levels (Table 1), while the 2-fold increase in serum sTfR levels reflects a strong iron uptake by the erythroid tissue $^{32}$ (Table 2). This high acquisition of iron by the erythroid cells in HA is paralleled by a decrease in body iron storage as indicated by the $40 \%$ decrease in serum ferritin levels (Table 2). The $56 \%$ increase $(P<.01)$ in serum ceruloplasmin levels (Table 2) also indicates a strong overall iron mobilization and further supports the concept of an increased iron transfer to the erythropoietic compartment during HA.

\section{Elevated iron incorporation in the erythropoietic tissue is associated with an alteration of iron homeostasis in skeletal muscle}

Concurrent with the blood analyses, the response to HA of the muscle proteins involved in iron metabolism was explored in biopsy samples. First, by means of an ELISA assay, we observed that the L-ferritin subunit, but not the $\mathrm{H}$-ferritin subunit, was found to decrease $(P<.05)$ in HA (Figure 1A). In agreement with the lower level of L-ferritin, which is related to intracellular iron deposits, ${ }^{33}$ muscle total iron content was also found to be reduced $(P<.05 ;$ Figure 1B). These findings suggest that iron mobilization does occur at the muscle level. In addition, Western blot analysis with $\alpha$-tubulin normalization showed a $50 \%$ decrease $(P<.01)$ in the expression of TfR protein in HA (Figure 2A-B). This response might be associated with changes in TfR mRNA content, as a small, albeit nonsignificant decrease in the TfR mRNA level was found

\section{Table 1. Blood gases, hemoglobin, and serum EPO}

\begin{tabular}{lcc}
\hline & SL, mean \pm SD & HA (4559 m), mean \pm SD \\
\hline Hemoglobin, g/L-1 & $147 \pm 8$ & $170 \pm 13^{*}$ \\
$\mathrm{PaCO}_{2}, \mathrm{mmHg}$ & $40.6 \pm 2.1$ & $29.1 \pm 2.0^{*}$ \\
$\mathrm{PaO}_{2}, \mathrm{mmHg}$ & $105.7 \pm 15.7$ & $50.3 \pm 3.0^{*}$ \\
$\mathrm{SaO}_{2}, \%$ & $98.5 \pm 0.6$ & $83.6 \pm 2.9^{\star}$ \\
Arterial O$_{2}$ content, $\mathrm{mL} / \mathrm{L}^{-1}$ & $199 \pm 10$ & $192 \pm 19$ \\
$\mathrm{PfvO}_{2}, \mathrm{mmHg}$ & $37.9 \pm 6.3$ & $35.0 \pm 5.5$ \\
$\mathrm{EPO}, \mathrm{mlU} / \mathrm{mL}^{-1}$ & $8.7 \pm 3.7$ & $12.2 \pm 3.0^{*}$ \\
\hline
\end{tabular}

The values are mean \pm SD for 9 participants. $\mathrm{PaCO}_{2}$ and $\mathrm{PaO}_{2}$ indicate arteria carbon dioxide and oxygen pressures, respectively; $\mathrm{SaO}_{2}$, arterial oxygen saturation; $\mathrm{PfvO}_{2}$, femoral venous oxygen pressure. Arterial and venous samples were obtained under normoxic conditions at SL and during exposure to HA (4559 m) for 7 to 9 days. The calculation of statistical differences from normoxia was performed using the Wilcoxon test.

${ }^{\star} P<.01$. 
Table 2. Serum iron parameters

\begin{tabular}{lcc}
\hline & Sea level & HA (4559 m) \\
\hline sTfR, $\mathrm{nmol} / \mathrm{L}^{-1}$ & $12.9 \pm 2.4$ & $26.4 \pm 9.3^{*}$ \\
Ferritin, $\mu \mathrm{g} / \mathrm{L}^{-1}$ & $98.4 \pm 75.2$ & $57.3 \pm 70.3^{*}$ \\
Transferrin, $\mathrm{g} / \mathrm{L}^{-1}$ & $2.3 \pm 0.3$ & $2.9 \pm 0.5^{\star}$ \\
Iron saturation of transferrin, \% & $17 \pm 13$ & $6 \pm 8 \dagger$ \\
Ceruloplasmin, $\mathrm{g} / \mathrm{L}^{-1}$ & $0.179 \pm 0.031$ & $0.278 \pm 0.052^{*}$ \\
\hline
\end{tabular}

The values are mean \pm SD for 9 participants. STfR indicates soluble transferrin receptor. Venous samples were obtained under normoxic conditions at SL and during exposure to HA (4559 m) for 7 to 9 days. The calculation of statistical differences from normoxia was performed using the Wilcoxon test.

${ }^{\star} P<.01$.

$\dagger P=.06$.

(Figure 2C). Because the parallel decrease of both TfR and ferritin is an unexpected finding, 5,6 the molecular mechanisms involved in the control of ferritin and TfR expression in HA were further analyzed. First, the binding activity of the IRPs, evaluated by means of RNA bandshift analysis, revealed a relatively high interindividual variability but no significant modifications in HA (Figure 3A-B). As human IRP1 and IRP2 comigrate in the RNA bandshift assay used in the present study, the unchanged combined activity of the 2 IRPs does not exclude the possibility that either IRP1 or particularly IRP2, thought to be a key regulator of cellular iron homeostasis, ${ }^{34,35}$ is actually modified during high-altitude exposure. However, the limited amount of material prevented us to perform supershift assay or immunoblotting analyses to assess this possibility. Nevertheless, in the present study, the IRP's system does not seem to be the main regulatory pathway accounting for the observed decreases in either TfR or ferritin in HA. Second, the mRNA levels of both HIF- $1 \alpha$ and HIF- $2 \alpha$ increased by 2.3 -fold $(P<.05)$ and 3.3 -fold $(P<.05)$, respectively, in HA (Figure 4$)$. The finding of low TfR levels with high HIF-1 mRNAs in HA contrasts with data obtained in cell cultures where TfR mRNA is up-regulated in hypoxia when HIF-1 accumulates, ${ }^{3,4}$ thereby suggesting that the HIF-1-mediated pathway plays a somewhat different role in humans under the present experimental conditions. Finally, the analysis of ferroportin mRNA levels revealed a large variability between individuals but also a more than 6-fold increase $(P=.05)$ upon exposure to HA (Figure 5). This result might indicate an increased muscle iron export in HA, as ferroportin is known to be the major or only cell iron exporter. ${ }^{36}$ In summary, the alteration of muscle iron homeostasis observed after 7 to 9 days of exposure to HA suggests that lower iron incorporation in muscle and/or higher iron export from muscle occurs in HA.

\section{Myoglobin content is decreased at HA}

To gain further insights into the interactions between iron and oxygen regulation in skeletal muscle, myoglobin expression in muscle was also assessed by $2 \mathrm{D}$ gel electrophoresis followed by fluorescent staining (Figure 6A). Human myoglobin is characterized by 3 isoforms. ${ }^{17}$ The main isoform (isoform 1) that, in control conditions, amounts to $75 \%$ to $80 \%$ of the total, was decreased by $35 \%(P<.01)$ in HA (Figure 6B), while the isoform $2(15 \%-20 \%$ of the total) and isoform 3 (5\% of the total) were decreased by $30 \%$ $(P<.05)$ and $27 \%(P=.05)$, respectively, in HA (Figure 6C-D). In summary, the perturbations of muscle iron metabolism occurring at high altitude are associated with a decrease in myoglobin levels, suggesting an alteration of muscle oxygen homeostasis.

\section{Discussion}

Iron plays a central role in protecting the organism from hypoxemia, as it is incorporated in the newly synthesized hemoglobin throughout erythropoiesis. The findings of the present study show that, in humans, this essential adaptive response to the early phase of hypoxia (7-9 days) is associated with a net loss in the iron content of skeletal muscle, as indicated by the down-regulation of the iron proteins ferritin and TfR, and the possible up-regulation of ferroportin in muscle. It is also possible that, in turn, an insufficient iron supply accounts for the lower myoglobin expression observed during hypoxia.

To the best of our knowledge, the molecular physiology of iron metabolism in human skeletal muscle has received very little attention, which is surprising as it contains $10 \%$ to $15 \%$ of total body iron. ${ }^{13}$ Skeletal muscle plays a functional role in oxygen storage, transport, and use, and iron is a key component of myoglobin and the heme groups of cytochromes. By examining humans exposed to 7 to 9 days of severe hypoxia, the present study provides new insight into the molecular aspects of iron-oxygen interactions in response to altered systemic iron metabolism secondary to hypoxia-induced erythropoiesis.

The coexistence of functional iron deficiency and hypoxia during prolonged exposure to high altitude makes it difficult to distinguish the role of hypoxia per se from the effect of increased erythropoiesis on muscle iron metabolism. The following discussion will therefore consider the potential effects of both stimuli.

A first possible interpretation is to assume that the changes in muscle iron homeostasis observed at high altitude are primarily due to a direct effect of hypoxia. In order to address this question, it is
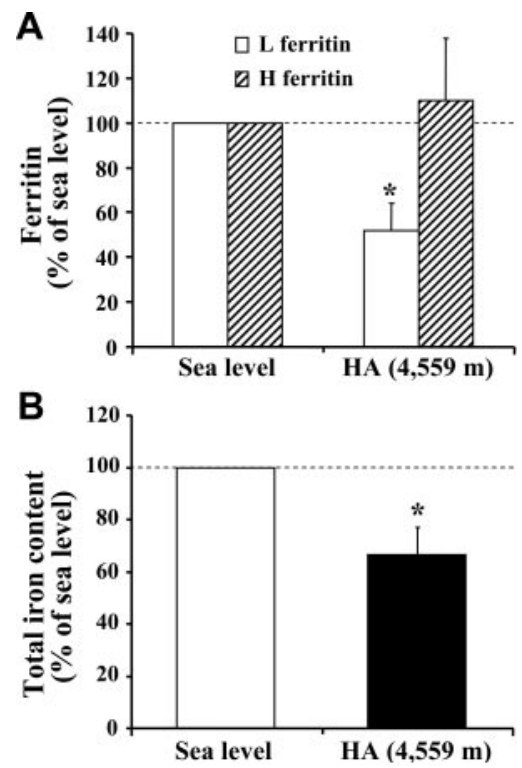

Figure 1. High-altitude exposure decreases L-ferritin and total iron content in skeletal muscle. The changes in ferritin and iron content in muscle biopsies obtained from vastus lateralis under normoxic conditions at SL and during exposure to HA (4559 $\mathrm{m}$ ) for 7 to 9 days are shown. (A) Ferritin $\mathrm{H}$ and $\mathrm{L}$ subunit contents. The amount of $\mathrm{H}$ and $\mathrm{L}$ subunits in extracts of muscle biopsies was determined by ELISA using monoclonal antibodies against the corresponding recombinant human ferritin subunits. The $\mathrm{H}$ - and $\mathrm{L}$-ferritin contents are expressed relative to the value at $\mathrm{SL}$, which was arbitrarily defined as 100 . The values are mean \pm SE of 4 determinations in 9 participants. (B) Total iron content. The determination was performed by atomic absorption spectrometry. The total muscle iron content (measured in $\mu \mathrm{g} / 100 \mathrm{mg}$ dry weight) is expressed relative to the value at SL, which was arbitrarily defined as 100 . The values are means \pm SE for 9 participants. The calculation of statistical differences from normoxia was performed using the Wilcoxon test. ${ }^{*} P<.05$. 

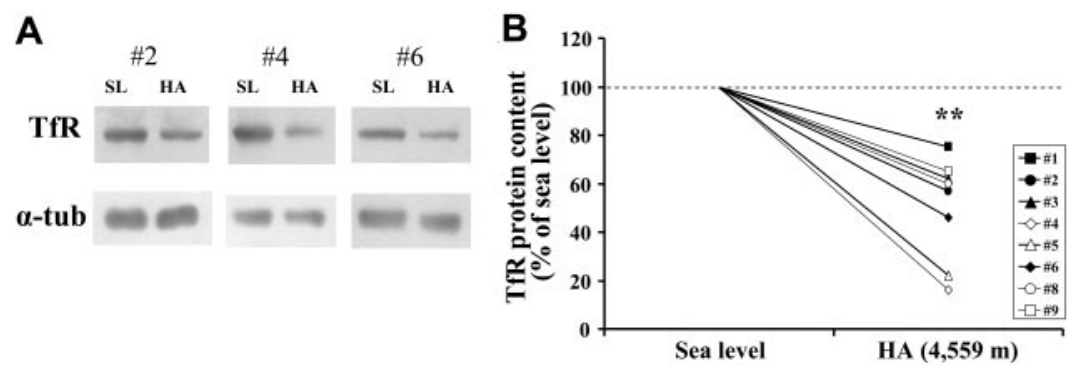

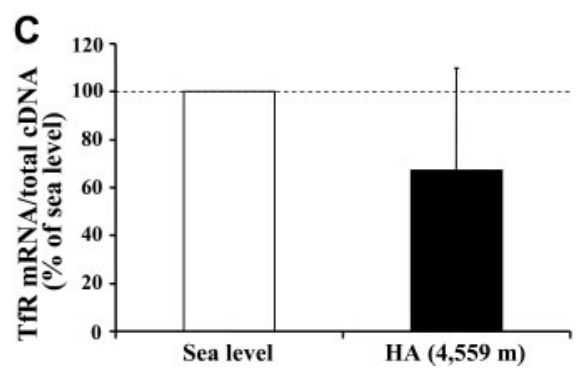

Figure 2. High-altitude exposure decreases TfR protein content in skeletal muscle. The changes in TfR expression in muscle biopsies obtained from vastus lateralis under normoxic conditions at SL and during exposure to HA (4559 m) for 7 to 9 days are shown. (A) Equal amounts of proteins from extracts of muscle biopsies were loaded onto SDS polyacrylamide gels and immunoblotted with antibodies against TfR. The blots were reprobed with an antibody against $\alpha$-tubulin ( $\alpha$-tub) as a loading control. Antigens were visualized by chemiluminescence. Extracts of participant nos. 2, 4, and 6 were processed separately. (B) Densitometric quantification of immunoblot analysis of TfR protein content at SL and during HA. Individual changes for all study participants but no. 7, whose sample could not be analyzed because of technical reasons, are shown. (C) TfR mRNA levels. The TfR mRNA content is normalized to total cDNA content and expressed relative to the value at SL, which was arbitrarily defined as 100 . The values are means \pm SE for 9 participants. The calculation of statistical differences from normoxia was performed using the Wilcoxon test. ** $P<.01$.

necessary to evaluate the degree of hypoxia experienced at the systemic level and at the muscle level by subjects acclimatized to HA for 7 to 9 days. At the systemic level, despite permanently lower $\mathrm{PaO}_{2}$ and $\mathrm{SaO}_{2}$ at high altitude, the oxygen-carrying capacity of blood normalizes as the result of an increase in hemoglobin concentration that is mediated by 2 cumulative mechanisms: (1) decreased plasma volume; ${ }^{37}$ and (2) the synthesis of new hemoglobin molecules. At the muscle level, it is known that oxygen pressure is very low even in a normoxic environment ${ }^{38}$ and, although we did not determine muscle intracellular $\mathrm{PO}_{2}$, the finding of unchanged femoral venous $\mathrm{PO}_{2}$ levels indicates that the oxygenation of the muscle microcirculation was well preserved at high altitude, even if we cannot exclude the possibility that the decrease in myoglobin levels at high altitude can in turn restrict muscle oxygen content.

A second possible interpretation is to assume that the highaltitude changes in muscle iron homeostasis are primarily due to an iron deficiency related to increased erythropoiesis secondary to hypoxia, rather than to a direct effect of hypoxia itself. At a systemic level, accelerated erythropoiesis, bone marrow iron uptake, and body iron mobilization at high altitude are respectively revealed by high serum EPO and sTfR levels, low serum ferritin levels, and high serum ceruloplasmin levels. At a muscle level, our results strongly suggest that muscle tissue loses iron at high
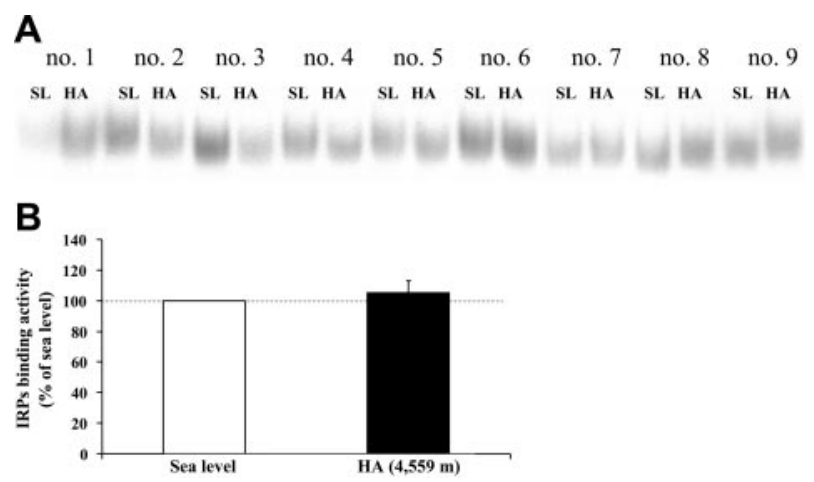

Figure 3. The activity of IRPs in skeletal muscle is not altered during high-altitude exposure. RNA bandshift analysis of IRPs activity in muscle biopsies obtained from vastus lateralis under normoxic conditions at $S L$ and during exposure to HA (4559 m) for 7 to 9 days. Cytosolic extracts were incubated with an excess of a ${ }^{32} \mathrm{P}$-labeled IRE probe, and the RNA-protein complexes were separated on nondenaturing polyacrylamide gels. (A) A typical bandshift representative of 1 experiment performed with extracts of the 9 participants of the present study. (B) Quantification of IRP binding activity obtained from 3 independent determinations and expressed relative to the value at SL, which was arbitrarily defined as 100 . The values are means \pm SE for 9 participants. altitude: there is a decrease in L-ferritin, total iron, and TfR levels, an increase in ferroportin mRNA, and a decrease in myoglobin content. The concomitance of these systemic and muscular events leads us to suggest that the iron requirement of erythropoietic tissue plays an important and possibly predominant role in muscle iron mobilization at high altitude. This hypothesis is supported by preliminary results showing that prolonged treatment with low doses of recombinant human EPO (90 000 IU over 14 weeks) at sea level (ie, under normoxic conditions) decreases muscle L-ferritin in healthy humans (G.C., P.R., and C.L., unpublished data, October 2006). Further evidence that increased erythropoiesis may deplete tissue iron comes from a recent study indicating that transgenic mice overexpressing EPO have virtually no iron in the teeth and other tissues (M. Gassmann, oral communication, February 2005), as it is also shown by the whiteness of their teeth in comparison with the yellow teeth of the wild-type animals. ${ }^{39}$

With respect to the systemic signal triggering muscle iron mobilization at high altitude, the involvement of hepcidin is suggested. This liver-derived peptide, which seems to be the key regulator of iron homeostasis, 8,40 binds to ferroportin at the cell surface, and triggers ferroportin internalization and degradation. ${ }^{41}$ As it has previously been shown that decreased iron availability down-regulates hepcidin production by the liver, ${ }^{42}$ hepcidin levels may be reduced in subjects exposed to high altitude, which may increase the ferroportin localized on muscle cell membrane, and thus enhance the iron export already favoured by increase in ferroportin mRNA levels observed in subjects

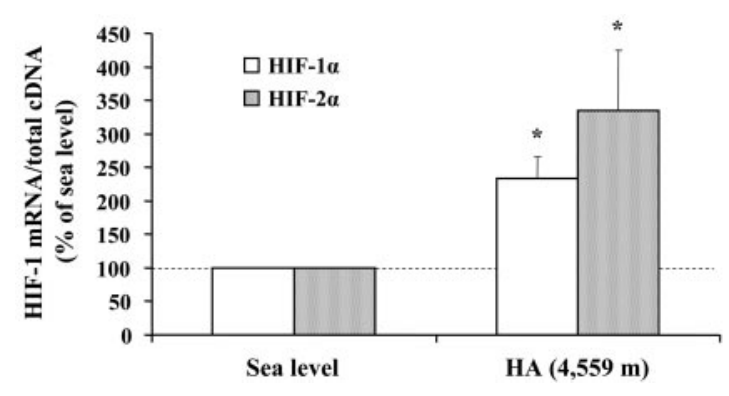

Figure 4. High-altitude exposure is associated with an increase in skeletal muscle HIF-1 mRNA levels. The changes in HIF- $1 \alpha$ and HIF-2 $\alpha$ mRNA levels in muscle biopsies obtained from vastus lateralis under normoxic conditions at SL and during exposure to HA (4559 m) for 7 to 9 days are shown. The target mRNA content is normalized to total cDNA content and expressed relative to the value at SL, which was arbitrarily defined as 100 . The values are means \pm SE for 9 subjects. The calculation of statistical differences from normoxia was performed using the Wilcoxon test. ${ }^{*} P<.05$. 


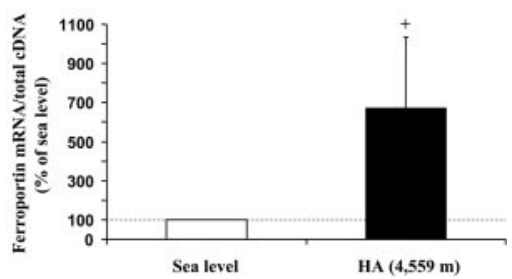

Figure 5. High-altitude exposure is associated with an increase in skeletal muscle ferroportin mRNA levels. The changes in ferroportin mRNA levels in muscle biopsies obtained from vastus lateralis under normoxic conditions at SL and during exposure to HA (4559 m) for 7 to 9 days are shown. The target mRNA content is normalized to total cDNA content and expressed relative to the value at SL, which was arbitrarily defined as 100 . The values are means \pm SE for 9 participants. The calculation of statistical differences from normoxia was performed using the Wilcoxon test. $+P=.05$.

exposed to high altitude. It can also be further speculated that hemojuvelin may be involved in such a setting, as it is highly expressed in muscle tissue, ${ }^{43}$ and its soluble form might interact with the protein complex on the surface of the hepatocytes regulating hepcidin expression.

Regarding the molecular mechanism(s) associated with decreased muscle iron content, our tissue analyses revealed an unexpected response at high altitude (ie, a parallel decrease in muscle ferritin and TfR), which conflicts with the classic IRE/IRPmediated posttranscriptional regulation that usually leads to the coordinate but inverse expression of the 2 proteins. ${ }^{5,6}$ The finding of unchanged IRP binding activity at high altitude despite altered iron homeostasis suggests that IRP-independent mechanisms may be involved. It is worth noting that the expression of genes encoding the proteins of iron metabolism is subject to additional regulatory mechanisms, as shown by the fact that the expression of IRP-regulated genes can bypass the control of IRPs. ${ }^{44-47}$ An alternative molecular-based explanation of the present data comes from the recent demonstration that ferritin iron release and subsequent proteasomal degradation occur in cells overexpressing ferroportin. ${ }^{48}$

However, although muscle ferritin down-regulation in subjects exposed to high altitude is consistent with the indications of iron loss, the molecular mechanisms underlying TfR down-regulation are more difficult to explain. In addition to iron-mediated posttranscriptional regulation, TfR expression is also subject to HIF-1mediated transcriptional control, but the finding of increased HIF-1 mRNA levels during high-altitude exposure does not offer a satisfactory molecular basis for the down-modulation of TfR because evidence from cell culture studies indicates that HIF-1 induces TfR transcription under hypoxic conditions. ${ }^{3,4}$ However, whatever the mechanism may be, when considering the role of ferroportin in iron export and TfR in iron uptake, higher ferroportin mRNA levels and lower TfR expression are consistent with the loss of iron from the muscle cells of subjects exposed to high altitude.

Another important question is whether the muscle iron loss at high altitude is biologically relevant to muscle function. The concomitant decrease in myoglobin expression and muscle iron proteins suggests that altered iron homeostasis may have an impact on myoglobin and therefore on muscle oxygen homeostasis. Given the functional role of myoglobin as a protein facilitating oxygen storage and diffusion, ${ }^{14,15}$ as well as other emerging roles such as nitric oxide and reactive oxygen species scavenging, ${ }^{49}$ our data highlight the potential importance of the regulation of iron distribution to muscle oxygen homeostasis. These observations conflict with the view that myoglobin levels increase during prolonged hypoxia ${ }^{17,18,20,21}$ in order to maintain muscle oxygen homeostasis in the low-oxygen environment, as well as with the recent demonstration that myoglobin expression is induced by severe hypoxia in fish muscle and nonmuscle tissues. ${ }^{16}$ One reason for this discrepancy could be the time spent in hypoxia, which was much shorter in the present study. In previous studies of chronic hypoxia, ${ }^{17,18,20}$ it is likely that red blood cell mass and the rate of erythropoiesis had both stabilized, thus allowing the availability of more iron for myoglobin synthesis in muscle than during the early phase of altitude acclimatization.

The connection between iron homeostasis and oxygen metabolism has been previously highlighted by molecular and cellular studies showing the role of iron in the modulation of HIF-1 activity $^{1,50}$ and, conversely, the role of oxygen levels in regulating IRP activities. ${ }^{34}$ Moreover, iron homeostasis is disrupted when HIF-1 is absent ${ }^{2}$ or up-regulated. ${ }^{51}$ The findings of the present study provide new insights into the interactions between iron distribution
Figure 6. High-altitude exposure results in a decrease in myoglobin protein expression in skeletal muscle. The individual variations in myoglobin levels in muscle biopsies obtained from vastus lateralis under normoxic conditions at $S L$ and during exposure to HA (4559 m) for 7 to 9 days are shown. Myoglobin content in extracts of muscle biopsies was analyzed by $2 \mathrm{D}$ gel electrophoresis followed by SYPRO Ruby staining. The quantification of the separated isoforms was performed by the Image Quant software. The values are given in arbitrary units (AU). Due to technical reasons, the analysis of 1 sample (no. 5) could not be performed. (A) A typical 2D gel electrophoresis spot image, showing the 3 myoglobins isoforms, is presented for participant no. 7 at SL and during HA. The individual quantitative changes for all study participants relative to myoglobin isoform 1 (main isoform) (B), myoglobin isoform 2 (C), and myoglobin isoform 3 (D) (isoforms 2 and 3 being expressed at lower levels also under normoxic conditions) are presented. The data are representative of 3 independent experiments. The calculation of statistical differences from normoxia was performed using the Wilcoxon test. ${ }^{\star \star} P<.01 ;{ }^{*} P<.05 ;+P=.05$
A

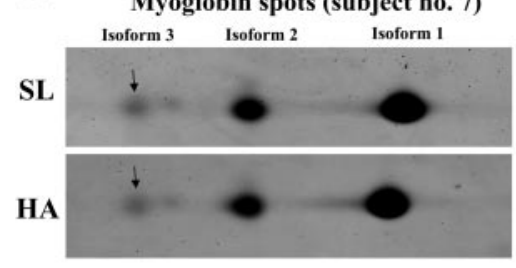

C

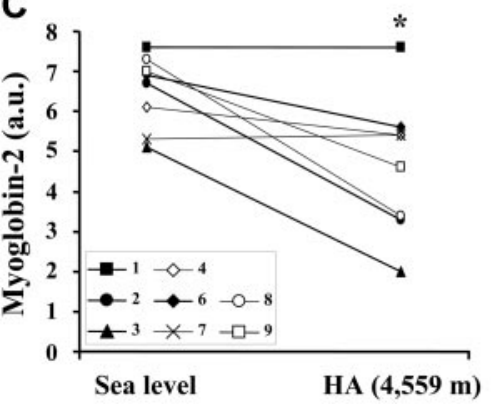

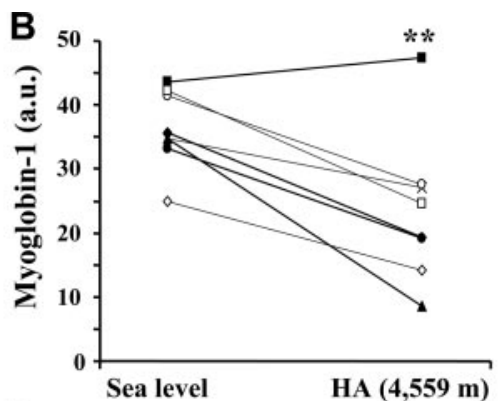

D

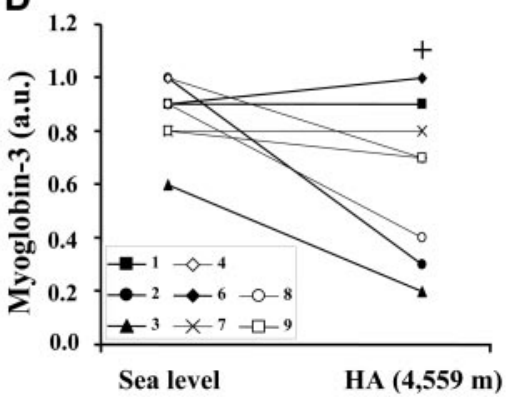


and oxygen homeostasis at the whole-body level in humans by suggesting that there is a net loss of iron from skeletal muscle during the systemic iron redistribution associated with high bone marrow iron uptake following hypoxia-enhanced erythropoiesis. We suggest that, under prolonged exposure to hypoxia, the beneficial process of hemoglobin production initiates an unfavourable response in skeletal muscle, and that the 2 events are linked by changes in iron trafficking. The possibility of impaired muscle oxygen homeostasis due to iron-related mechanisms (and probably the reduction of the capacity for physical exercise) may have important clinical implications, because both hypoxic conditions and disturbed erythropoiesis are common in clinical settings.

\section{Acknowledgments}

The authors thank Kristina Møller Kristensen for excellent technical assistance and Paola Dongiovanni for help with the determination of total iron content, as well as Marinela Ripamonti for stimulating discussions.
Supported by research funding from the Novo Nordisk Foundation and the Danish Science Foundation to C.L. This study was also supported in part by research funding from EvK2CNR Committee to C.G. and P.C. and from MIUR to G.C.

\section{Authorship}

Author contributions: P.R. designed research, performed research, collected data, analyzed data, and wrote the paper; G.C. designed research, contributed vital new reagents or analytical tools, collected data, analyzed data, and wrote the paper; C.G., F.B., H.P., A.V., P.S., P.C. and S.M. contributed vital new reagents or analytical tools and collected data; J.A.L.C. and C.L. designed research, performed research, collected data and analyzed data. P.R and G.C. contributed equally to this work.

Conflict-of-interest statement: The authors declare no competing financial interests.

Correspondence: Gaetano Cairo, University of Milan School of Medicine, Istituto di Patologia Generale, Via Mangiagalli 31, 20133 Milano, Italy; e-mail: gaetano.cairo@unimi.it.

\section{References}

1. Semenza GL. HIF-1: mediator of physiological and pathophysiological responses to hypoxia. J Appl Physiol. 2000;88:1474-1480.

2. Yoon D, Pastore YD, Divoky V, et al. Hypoxiainducible factor-1 deficiency results in dysregulated erythropoiesis signaling and iron homeostasis in mouse development. J Biol Chem. 2006; 281:25703-25711.

3. Lok CN, Ponka P. Identification of a hypoxia response element in the transferrin receptor gene. J Biol Chem. 1999;274:24147-24152.

4. Tacchini L, Bianchi L, Bernelli-Zazzera A, Cairo G. Transferrin receptor induction by hypoxia. HIF1-mediated transcriptional activation and cellspecific post-transcriptional regulation. J Biol Chem. 1999;274:24142-24146.

5. Cairo G, Pietrangelo A. Iron regulatory proteins in pathobiology. Biochem J. 2000;352 Pt 2:241-250.

6. Hentze MW, Kuhn LC. Molecular control of vertebrate iron metabolism: mRNA-based regulatory circuits operated by iron, nitric oxide, and oxidative stress. Proc Natl Acad Sci U S A. 1996;93: 8175-8182.

7. Donovan A, Roy CN, Andrews NC. The ins and outs of iron homeostasis. Physiology (Bethesda). 2006;21:115-123.

8. Cairo G, Bernuzzi F, Recalcati S. A precious metal: iron, an essential nutrient for all cells. Genes \& Nutrition. 2006;1:25-40.

9. Ganz T, Nemeth E. Regulation of iron acquisition and iron distribution in mammals. Biochim Biophys Acta. 2006;1763:690-699.

10. Reynafarje C, Lozano R, Valdivieso J. The polycythemia of high altitudes: iron metabolism and related aspects. Blood. 1959;14:433-455.

11. Robach P, Fulla Y, Westerterp KR, Richalet JP. Comparative response of EPO and soluble transferrin receptor at high altitude. Med Sci Sports Exerc. 2004;36:1493-1498.

12. Richalet JP, Souberbielle JC, Antezana AM, et al. Control of erythropoiesis in humans during prolonged exposure to the altitude of $6,542 \mathrm{~m}$. Am J Physiol. 1994;266:R756-R764.

13. Boulton FE. The myoglobin content of human skeletal muscle. Br J Haematol. 1973;25:281.

14. Wittenberg JB, Wittenberg BA. Myoglobin function reassessed. J Exp Biol. 2003;206:2011-2020

15. Ordway GA, Garry DJ. Myoglobin: an essential hemoprotein in striated muscle. J Exp Biol. 2004; 207:3441-3446.
16. Fraser J, de Mello LV, Ward D, et al. Hypoxia-inducible myoglobin expression in nonmuscle tissues. Proc Natl Acad Sci U S A. 2006;103:29772981.

17. Gelfi C, De Palma S, Ripamonti M, et al. New aspects of altitude adaptation in Tibetans: a proteomic approach. FASEB J. 2004;18:612-614.

18. Reynafarje B. Myoglobin content and enzymatic activity of muscle and altitude adaptation. J Appl Physiol. 1962;17:301-305.

19. Terrados N, Jansson E, Sylven C, Kaijser L. Is hypoxia a stimulus for synthesis of oxidative enzymes and myoglobin? J Appl Physiol. 1990;68: 2369-2372.

20. Vaughan BE, Pace N. Changes in myoglobin content of the high altitude acclimatized rat. Am J Physiol. 1956;185:549-556.

21. Hoppeler H, Vogt M. Muscle tissue adaptations to hypoxia. J Exp Biol. 2001;204:3133-3139.

22. Calbet JA, Lundby C, Sander M, Robach P, Saltin $B$, Boushel R. Effects of ATP-induced leg vasodilation on VO2 peak and leg $\mathrm{O} 2$ extraction during maximal exercise in humans. Am J Physiol Regu Integr Comp Physiol. 2006;291:R447-R453.

23. Cozzi A, Corsi B, Levi S, Santambrogio P, Biasiotto G, Arosio P. Analysis of the biologic functions of $\mathrm{H}$ - and L-ferritins in HeLa cells by transfection with siRNAs and cDNAs: evidence for a proliferative role of L-ferritin. Blood. 2004; 103:2377-2383.

24. Mullner EW, Neupert B, Kuhn LC. A specific mRNA binding factor regulates the iron-dependent stability of cytoplasmic transferrin receptor mRNA. Cell. 1989;58:373-382.

25. Cairo G, Tacchini L, Pogliaghi G, Anzon E, Tomasi A, Bernelli-Zazzera A. Induction of ferritin synthesis by oxidative stress: transcriptional and post-transcriptional regulation by expansion of the "free" iron pool. J Biol Chem. 1995;270:700703.

26. Gelfi C, Vigano A, Ripamonti M, et al. The human muscle proteome in aging. J Proteome Res. 2006;5:1344-1353.

27. Chomczynski $P$, Sacchi N. Single-step method of RNA isolation by acid guanidinium thiocyanatephenol-chloroform extraction. Anal Biochem. 1987:162:156-159.

28. Pilegaard H, Ordway GA, Saltin B, Neufer PD. Transcriptional regulation of gene expression in human skeletal muscle during recovery from ex- ercise. Am J Physiol Endocrinol Metab. 2000;279: E806-E814.

29. Pilegaard H, Saltin B, Neufer PD. Exercise induces transient transcriptional activation of the PGC-1alpha gene in human skeletal muscle. J Physiol. 2003;546:851-858.

30. Lundby C, Nordsborg N, Kusuhara K, Kristensen KM, Neufer PD, Pilegaard H. Gene expression in human skeletal muscle: alternative normalization method and effect of repeated biopsies. Eur J Appl Physiol. 2005;95:351-360.

31. Pietrangelo A, Rocchi E, Ferrari A, Ventura E, Cairo G. Regulation of hepatic transferrin, transferrin receptor and ferritin genes in human siderosis. Hepatology. 1991;14:1083-1089.

32. Beguin Y. The soluble transferrin receptor: biological aspects and clinical usefulness as quantitative measure of erythropoiesis. Haematologica. 1992;77:1-10.

33. Levi S, Santambrogio P, Cozzi A, et al. The role of the L-chain in ferritin iron incorporation: studies of homo and heteropolymers. J Mol Biol. 1994;238: 649-654.

34. Meyron-Holtz EG, Ghosh MC, Iwai K, et al. Genetic ablations of iron regulatory proteins 1 and 2 reveal why iron regulatory protein 2 dominates iron homeostasis. EMBO J. 2004;23:386-395.

35. Recalcati S, Alberghini A, Campanella A, et al. Iron regulatory proteins 1 and 2 in human monocytes, macrophages and duodenum: expression and regulation in hereditary hemochromatosis and iron deficiency. Haematologica. 2006;91:303310.

36. McKie AT, Marciani P, Rolfs A, et al. A novel duodenal iron-regulated transporter, IREG1, implicated in the basolateral transfer of iron to the circulation. Mol Cell. 2000;5:299-309.

37. Pugh LG. Blood volume and haemoglobin concentration at altitudes above $18,000 \mathrm{ft}$. (5500 m). J Physiol. 1964;170:344-354

38. Richardson RS, Noyszewski EA, Kendrick KF Leigh JS, Wagner PD. Myoglobin $\mathrm{O}_{2}$ desaturation during exercise. Evidence of limited $\mathrm{O}_{2}$ transport. J Clin Invest. 1995;96:1916-1926.

39. Heinicke K, Baum O, Ogunshola OO, et al. Excessive erythrocytosis in adult mice overexpressing erythropoietin leads to hepatic, renal, neuronal, and muscular degeneration. Am J Physiol Regul Integr Comp Physiol. 2006;291:R947R956. 
40. Ganz T, Nemeth E. Iron imports, IV: hepcidin and regulation of body iron metabolism. Am J Physiol Gastrointest Liver Physiol. 2006;290:G199-G203.

41. Nemeth E, Tuttle MS, Powelson J, et al. Hepcidin regulates cellular iron efflux by binding to ferroportin and inducing its internalization. Science. 2004;306:2090-2093.

42. Nicolas G, Chauvet C, Viatte L, et al. The gene encoding the iron regulatory peptide hepcidin is regulated by anemia, hypoxia, and inflammation. J Clin Invest. 2002;110:1037-1044.

43. Papanikolaou G, Samuels ME, Ludwig EH, et al. Mutations in HFE2 cause iron overload in chromosome 1q-linked juvenile hemochromatosis. Nat Genet. 2004;36:77-82.

44. Seiser C, Teixeira S, Kuhn LC. Interleukin-2-de- pendent transcriptional and post-transcriptional regulation of transferrin receptor mRNA. J Bio Chem. 1993;268:13074-13080.

45. Abboud S, Haile DJ. A novel mammalian ironregulated protein involved in intracellular iron metabolism. J Biol Chem. 2000;275:19906-19912.

46. Cairo G, Tacchini L, Pietrangelo A. Lack of coordinate control of ferritin and transferrin receptor expression during rat liver regeneration. Hepatology. 1998;28:173-178.

47. Wang J, Pantopoulos K. Conditional derepression of ferritin synthesis in cells expressing a constitutive IRP1 mutant. Mol Cell Biol. 2002;22: 4638-4651.

48. De Domenico I, Vaughn MB, Li L, et al. Ferroportin-mediated mobilization of ferritin iron precedes ferritin degradation by the proteasome. EMBO J. 2006;25:5396-5404.

49. Flogel U, Merx MW, Godecke A, Decking UK, Schrader J. Myoglobin: a scavenger of bioactive NO. Proc Natl Acad Sci U S A. 2001;98: 735-740.

50. Bianchi L, Tacchini L, Cairo G. HIF-1-mediated activation of transferrin receptor gene transcription by iron chelation. Nucleic Acids Res. 1999; 27:4223-4227.

51. Alberghini A, Recalcati S, Tacchini L, Santambrogio P, Campanella A, Cairo G. Loss of the von Hippel Lindau tumor suppressor disrupts iron homeostasis in renal carcinoma cells. J Biol Chem. 2005;280:30120-30128. 